\title{
Nanotechnology for developing countries
}

Nanotechnology covers the study, design, creation, synthesis, manipulation, and application of functional materials, devices, and systems through control of matter at the nanometer scale $\left(10^{-9} \mathrm{~m}\right)$, and the exploitation of novel phenomena and properties of matter at that scale. When matter is manipulated at a tiny scale of atoms and molecules, it exhibits novel phenomena and properties different from their bulk state. Thus, scientists are harnessing nanotechnology to create new, inexpensive materials, devices, and systems with unique properties.

Nanotechnology is often seen as one of the most promising cutting-edge technological breakthroughs of the modern times. It has the potential to be harnessed to address some of the most critical problems faced by nearly 5 billion people living in the developing world. Experts have identified 10 nanotechnology application areas most likely to have an impact in the developing world. These are, (1) Energy storage, production and conversion, (2) Agricultural productivity enhancement, (3) Water treatment and remediation, (4) Disease diagnosis and screening, (5) Drug delivery systems, (6) Food processing and storage, (7) Air pollution and remediation, (8) Construction, (9) Health monitoring, and (10) Vector and pest detection and control. Nanotechnology could provide solutions to problems in most of these key areas by developing cheap and easy-to-use nano materials, devices and systems. Many developing countries, including Sri Lanka, have already started using nanotechnology towards solving some of their socio-economic problems.

Nanotechnology can provide low cost and more efficient processes in energy generation, distribution, storage, and conversion where a lot of research and development is already taking place in laboratories in developing countries. In several developing countries, research teams working in the field of energy are focusing on nano-structured materials which can be used to build a new generation of low cost solar cells, hydrogen fuel cells and novel hydrogen storage systems. These technologies will help people in developing countries to avoid dependence on imported fossil fuels, and their adverse environmental impacts.

Research on low cost solar cells could offer an affordable photovoltaic energy generation for most developing countries, especially in the tropics. Nanotechnology based photovoltaics (cheaper and more efficient solar panels) and energy storage devices (batteries, fuel cells and supercapacitors) are the key areas for research and development in these countries. Low cost dye sensitized solar cells based on nano titania are near commercial production which can cut down the cost of electricity substantially. In Sri Lanka, there are several research groups working at the National Institute of Fundamental Studies and universities of Peradeniya, Jaffna, Kelaniya, Ruhuna and Wayamba, with the aim of developing low cost solar cells based on nano-titania and also low cost super capacitors based on high surface area nanomaterials for portable energy storage systems.

Under agriculture, nanotechnology scientists are developing a range of inexpensive nanotech applications to increase soil fertility and crop production, and help eliminate malnutrition which contributes to more than half the deaths of children under five in developing countries.

Nanocapsules, nanoparticles and even viral capsids are examples of uses to enahnce nutrient absorption by plants and the delivery of active ingredients to specific sites. The use of target-specific nanoparticles can reduce the damage to non-target plant tissues and the amount of chemicals released into the environment. Nanotechnology derived devices are also explored in the field of plant breeding and genetic transformation. These developments will greatly benefit the agricultural practices in developing countries.

Nanotech materials are being developed for slow release and efficient dosages of fertilizers for plants and of nutrients and medicines for livestock. Other agricultural developments include nano-sensors to monitor the health of crops and farm animals, and also magnetic nano-particles to remove soil contaminants. In contrast to conventional fertilizer use, which involves many tons of inputs, nanotechnology focuses on developing fertilizers required in small quantities. Scientists in developing countries are actively researching on nanofertilizers, for use in agriculture. Researchers at the Sri Lankan Institute of Nanotechnology (SLINTEC) have developed a slow-release nanofertilizer using urea coated hydroxyapatite nanoparticles for targeted delivery via nanohybrids. This has significantly reduced the amount of urea required for fertilization since it can be applied locally. Perhaps more impressively, these researchers have demonstrated that, with their novel finding, the rice yields can be significantly enhanced with $50 \%$ of the normal dosage of urea. SLINTEC is carrying out research in several focussed areas important for the national development. Nanofertilizer, insecticides and pesticides, nanofabrics and smart fabrics, nutraceuticals, grapheme and nano-titania are some of these R\&D areas. Value addition to local minerals available in Sri Lanka by purifying them and making them into nanoparticles with superior properties would enable the country to earn more foreign exchange while preserving the raw material. The possibility of converting Sri Lankan quartz sand into nano- 
silica is yet another example where funding for R\&D is needed.

One-sixth of the world's population lacks access to safe water supplies. More than one third of the population of rural areas in Africa, Asia, and Latin America has no clean water, while two million children die each year from water-borne diseases, such as diarrhoea, cholera, typhoid, and schistosomiasis, which result from a lack of adequate water sources and sanitation. Water filters made from nanomembranes and nano-clays are inexpensive, portable and purify, detoxify and desalinate water more efficiently than conventional bacterial and viral filters. These nanofilters, with the ability to remove several trace organic compounds, heavy metals and viruses at a lower energy cost than reverse osmosis, has found applications for the production of high quality drinking water in developed as well as developing nations. In Sri Lanka, several research groups are currently working on low cost nano-water filters using novel materials to remove bacteria and heavy metals. National Research Council and National Science Foundation have awarded a number of major research grants to develop these nanofiltration systems.

Under disease diagnosis and screening, possible nanotechnologies include the "lab-on-a-chip", which offers all the diagnostic functions of a medical laboratory, and other biosensors based on nano-sized tubes, wires, magnetic particles and semiconductor crystals (quantum dots). These inexpensive, hand-held diagnostic kits can detect the presence of several pathogens at once and could be used for wide-range screening in small peripheral clinics. Drug delivery systems including nano-capsules, dendrimers (tiny bush-like spheres made of branched polymers), and "buckyballs" (soccerball-shaped structures made of 60 carbon atoms) for slow and sustained drug release are being developed by research groups in many developing countries, including Sri Lanka. In the area of health monitoring, several nanomaterial-based low cost devices are being developed to keep track of daily changes in patients' physiological variables such as the levels of glucose, of carbon dioxide, and of cholesterol, without the need for drawing blood in a hospital setting. This way, patients suffering from diabetes would know at any given time the concentration of sugar in their blood; similarly, patients with heart diseases would be able to monitor their cholesterol levels easily. These low cost devices will benefit millions of patients in developing countries.

Nanomaterials that can destroy air pollutants using light have also been developed by research groups in several developing countries. These materials are able to detect toxic materials and any leaks to reduce fossil fuel emissions.

In the area of building construction, novel, nanoparticle incorporated-materials for cheaper and durable housing, including concrete, coatings and adhesives are being developed. Researchers at universities of Moratuwa and Peradeniya are currently working on developing nanoparticle incorporated low cost construction materials.

Many developing countries including India, China, Brazil and South Africa have invested R\&D funds on nanotechnology initiatives over the years. Other developing countries pursuing nanotechnology initiatives include Sri Lanka, Thailand, Philippines, Chile, and Argentina. In comparison to number of active researchers and funds invested by other developing countries for nanotechnology related research, Sri Lanka needs to do better.

M.A.K. Lakshman Dissanayake Email:makldis@yahoo.com 